\title{
Intereses e innovaciones para la Dehesa identificados por los agentes interesados
}

\author{
Motivations and innovations for the Dehesa identified \\ by land managers and other stakeholders \\ Moreno, G., Bertomeu, M., Cáceres, Y., Hernández-Esteban, A., Juárez, \\ E., López-Díaz, M.L., Pulido, F. \\ Grupo de Investigación Forestal - INDEHESA. Universidad de Extremadura. 10600 Plasencia, Cáceres \\ *Autor para correspondencia: gmoreno@unex.es
}

\section{Resumen}

En el marco del proyecto AGFORWARD (www.agforward.eu) que persigue promover las prácticas agroforestales en Europa como mecanismo de desarrollo rural, el Grupo de Trabajo de la Dehesa, formado por investigadores y actores interesados, ha trabajado a través de mesas de debate y encuestas en la identificación de los principales problemas y posibles soluciones para mejorar la productividad en la dehesa, su manejo y su conservación. Los resultados muestran que las dehesas pueden producir varios productos de alta calidad y proveer numerosos servicios ambientales. Sin embargo la baja rentabilidad comercial de la mayoría de las explotaciones de dehesa, junto con la progresiva degradación del suelo, pasto y arbolado, hacen peligrar la dehesa como sistema de explotación agroforestal. Las principales innovaciones propuestas son técnicas para la regeneración del arbolado, pastos permanentes ricos en leguminosas, cultivos forrajeros adaptados a la sombra, apoyo de las nuevas tecnologías al pastoreo racional y optimizar el aprovechamiento de los recursos forrajeros internos, y explorar la posibilidad de crear una marca para los productos de la dehesa.

\section{Summary}

In the framework of the AGFORWARD project (www.agforward.eu), which aims to promote agroforestry practices in Europe as a rural development mechanism, the Working Group of the Dehesa, made up of researchers and interested actors, has worked through debate tables and surveys in the identification of the main problems and possible solutions to improve the productivity in the dehesa, its management and its conservation. The results show that dehesas can produce several high quality products and provide numerous environmental services. However, the low commercial profitability of most dehesa farms, together with the progressive degradation of soil, pasture and trees, endanger the dehesa as an agroforestry exploitation system. The main innovations proposed are techniques for the regeneration of trees, permanent pastures rich in legumes, forage crops adapted to the shade, support of new technologies for rational grazing and optimizing the use of internal forage resources, and exploring the possibility of creating a brand for the products of the pasture.

Palabras clave: manejo integral, prácticas innovadoras, proceso participativo, productividad, sostenibilidad.

Keywords: integral management, innovative practices, participatory process, productivity, sustainability. 


\section{Introducción}

La orografía, la baja fertilidad edáfica y un clima difícil dificultan el desarrollo de la agricultura intensiva en muchas regiones Mediterráneas, donde el pastoreo extensivo ha sido el uso del suelo más común. Pero el pastoreo practicado actualmente generalmente impide la regeneración del arbolado (Pulido et al., 2010), lo que unido al empleo asociado del fuego, ha generado numerosos paisajes desarbolados en la regiones Mediterráneas (Rundel, 1998) y muchas otras partes del mundo (Asner et al., 2004, Ramankutty et al., 2008).

A esta dinámica global se han opuesto los sistemas silvopastorales, donde el pastoreo se ha practicado conservando cierta cobertura arbórea. Aquí se incluyen los bosques pastados, sabanas naturales tropicales, sabanas antrópicas (pastos arbolados) y prados con setos arbolados. Recientemente den Herder et al. (2016) han estimado en más de 15 millones de hectáreas la superficie ocupada por estos sistemas en Europa (EU-27). Y a nivel global éstos y otros sistemas agroforestales aún se mantienen de alguna manera en el $43 \%$ de las tierras manejadas agrícolamente del mundo, donde vive el 30\% de la población mundial (Zomer, 2014).

La conservación intencionada de los árboles en estos sistemas agroforestales responde a los múltiples productos que el hombre ha sabido aprovechar gracias a su presencia: madera, leña, frutos, forraje (ramoneo), materiales como el corcho, caza... Además la presencia del arbolado refuerza la biodiversidad y un gran número de servicios ambientales como el secuestro de carbono, la fertilidad del suelo, mejora del microclima, control de erosión y lixiviación y el bienestar animal (Jose, 2009; Moreno and Pulido, 2009).

Sin embargo, a pesar de estos beneficios múltiples de los sistemas agroforestales, la adopción de nuevos sistemas agroforestales en Europa no alcanza la relevancia que tiene en los países tropicales. Además la mayoría de los sistemas agroforestales tradicionales europeos, como los pastos arbolados, atraviesan por múltiples dificultades y van perdiendo progresivamente la importancia económica que tuvieron (Eichhorn et al., 2006; Gaspar et al., 2009; Rodriguez-Rigueiro et al., 2009).

El Proyecto de investigación AGFORWARD (www.agforward.eu), financiado por la Comisión Europea (VII Programa Marco) persigue promover las prácticas agroforestales en Europa como mecanismo de desarrollo rural. El proyecto se basa en la creación de una Red de Participación en Investigación y Desarrollo integrada por investigadores y actores interesados en los diferentes tipos de sistemas agroforestales europeos. Esta Red persigue identificar y evaluar innovaciones para aumentar la resiliencia de los sistemas agroforestales europeos.

En el marco de este proyecto en el Oeste Ibérico se ha creado el Grupo de Trabajo de la Dehesa (GTD). En este trabajo se presentan los resultados de los debates y encuestas realizadas dentro del GTD con el objetivo de:

1.- Identificar las principales dificultades y retos que deben afrontarse para la conservación y promoción de las dehesas.

2.- Elaborar y priorizar innovaciones para gestionar las dehesas de una forma 
más racional y sostenible económicamente.

3.- Identificar las principales iniciativas emprendidas por los participantes y organismos/organizaciones.

4.- Establecer una red de sitios experimentales para evaluar las innovaciones propuestas.

5.- Organizar actividades de diseminación incluyendo visitas a fincas con experiencias en curso.

\section{Material y métodos}

El primer paso del trabajo fue la creación del Grupo de Trabajo de la Dehesa (GTD) para lo que se contactó con investigadores, propietarios, agricultores, ganaderos, silvicultores (sobre todo relacionados con el corcho), representantes de organismos regionales y nacionales (incluidos técnicos de extensión agraria), representantes de organizaciones ambientales y de grupos de acción local y agentes de desarrollo rural de Extremadura y territorios cercanos ricos en dehesas (fundamentalmente sur de Castilla y León, oeste de Castilla la Mancha y norte de Andalucía). La creación del GTD se formalizó el 30 de mayo de 2014 en Plasencia (Escuela de Forestales de la Universidad de Extremadura) con 100 participantes, estando bien representados los diferentes agentes interesados antes mencionados.

Ese mismo día se organizaron mesas abiertas de debate, organizadas en 3 grupos y sesiones:

1.- Principales dificultades actuales de la dehesa.

2.- Propuestas de innovación para superar las dificultades y aumentar la sostenibilidad económica y ambiental de las dehesas.

3.- Estrategias y propuestas de investigación participativa con corresponsabilidad de investigadores y empresarios (propietarios). Las sesiones de debates fueron animadas por especialistas en dinámicas participativas (GEA S. COP.; http://geaweb.com). Los grupos participantes en las mesas de debate fueron flexibles y mezclados en las sucesivas sesiones de debate mantenidas. Cada participante señaló en una tarjeta las dos principales dificultades o desafíos que él/ella encuentran en sus fincas o en su experiencia en campo. Las tarjetas se discutieron una por una, para posteriormente ser agrupadas y ordenadas.

Al final de la jornada (o en los días siguientes) los participantes $(n=78)$ rellenaron una primera encuesta (cuestionario semi-estructurado presencial) para identificar su percepción en cuanto a las ventajas y desventajas productivas, de gestión, ambientales y socio-económicas de la dehesa frente a los pastos no arbolados. Una segunda encuesta, realizada on-line, fue lanzada para priorizar entre las 54 temáticas de investigación demandadas en la jornada de debate abierto por los componentes del GTD. A cada encuestado $(n=43)$ se le pedía identificar y ordenar por orden de prioridad las 10 temáticas más relevantes. Finalmente, en ambas encuestas, a los 
propietarios/responsables de explotaciones se les pedía que indicaran la disponibilidad para co-participar en ensayos concretos en sus explotaciones.

\section{Resultados y discusión}

\subsection{Dificultades para la Sostenibilidad Ambiental y Económica de la Dehesa}

En la Fig. 1 se presenta una visión general de la percepción de los aspectos positivos y negativos que los participantes expresan en la primera encuesta respecto a la dehesa ibérica. Específicamente, la estética del paisaje, la conservación de suelos, la modulación del microclima, la biodiversidad y el secuestro de carbono fueron los mejor valorados. Otros aspectos, como el bienestar animal, la regeneración del arbolado, la producción animal, la originalidad y el interés del sistema y el turismo también recibieron valoraciones muy positivas. Por el contrario, los encuestados tenían una percepción más negativa de los aspectos relativos a la gestión diaria y a la economía de los sistemas agroforestales, como los gravámenes de carácter administrativo, la falta de subsidios y subvenciones idóneas, dificultades de mecanización, los impuestos, el problema del flujo de caja, la necesidad de múltiples habilidades y los costes laborales.

Además de la percepción de los sistemas agroforestales expresada en el cuestionario, el debate abierto planteó una serie de preocupaciones sobre la productividad en la dehesa, su manejo y su conservación (Fig. 2), de las que cabe destacar:

- La falta de evaluaciones fiables sobre la rentabilidad en la dehesa.

- El estado sanitario de los árboles de la dehesa. El sobre-envejecimiento de los árboles de la dehesa y la falta de regeneración de éstos.

- La mala calidad de los suelos y los pastos de la dehesa. La baja productividad y la fuerte estacionalidad. Discrepancia entre los recursos de pastoreo y la carga animal.

- Los problemas de salud del ganado y re-infestación continua de tuberculosis (TB) a causa de las especies de caza mayor.

- El pago por servicios ambientales públicos que, presumiblemente, son importantes en las dehesas, pero que todavía no están cuantificados ni se aplican.

- La falta de gobernanza y comunicación con los organismos políticos.

- Ausencia de normativas adaptadas a usos múltiples y a los agrosistemas seminaturales así como a los pastizales arbolados extensivos.

\subsection{Propuestas de Innovación para la Sostenibilidad Ambiental y Económica de la Dehesa}

Durante la jornada de debate abierto el GTD identificó una larga lista de innovaciones que podrían ser de utilidad pero que necesitan de investigación antes de 


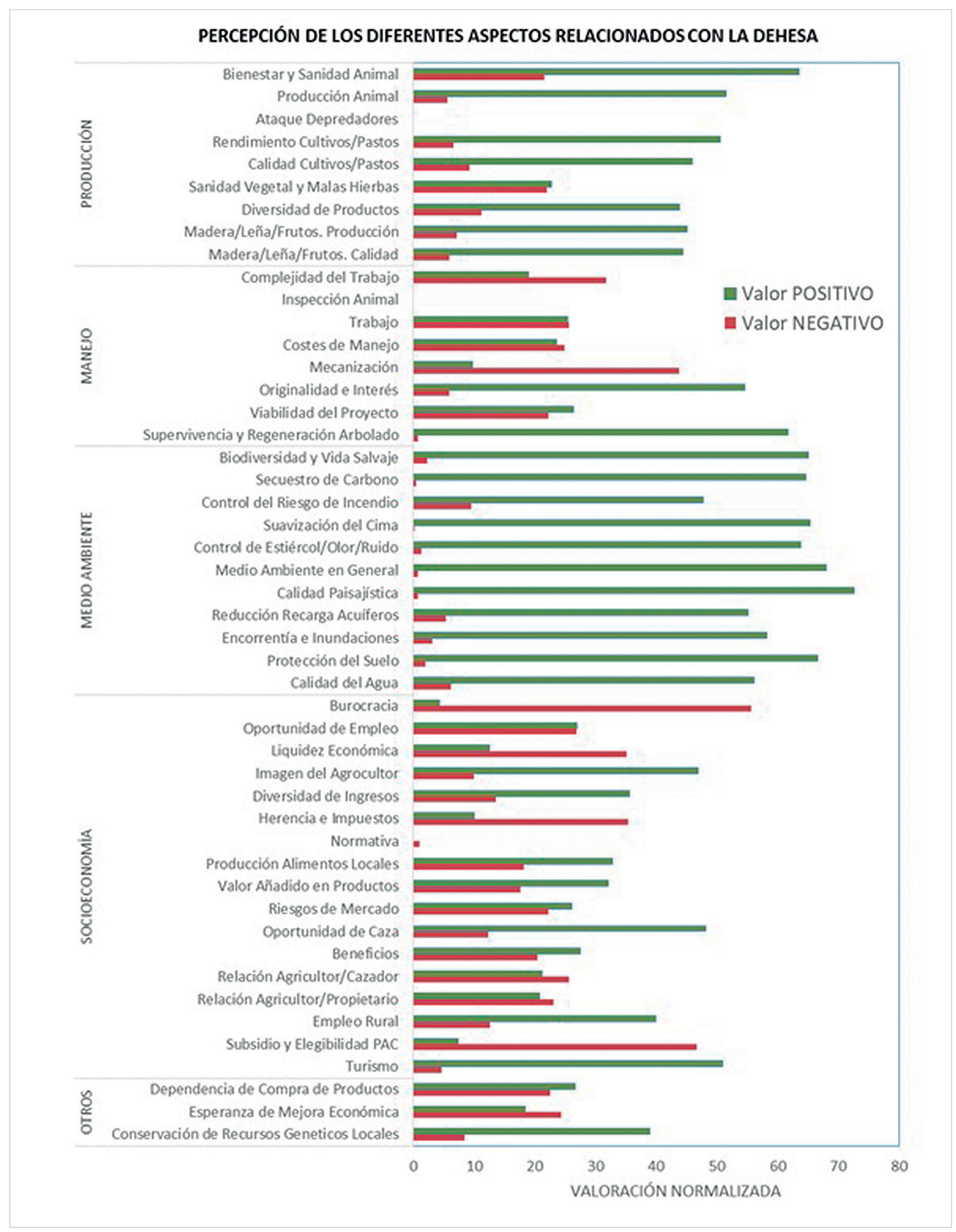

Figura 1. Valores positivos y negativos otorgados por los agentes interesados de la dehesa ibérica $(n=78$ encuestas) a 48 aspectos diferentes relacionados con los sistemas y prácticas agroforestales. Los 48 temas se agrupan en 5 bloques temáticos: Producción, manejo, medio ambiente, aspectos socio-económicos y otros aspectos relacionados con la calidad de vida. Cada encuestado priorizaba entre los aspectos positivos y negativos (hasta 5 en cada caso). A partir de las respuestas se calculó el valor final otorgado a cada tema, y los valores fueron normalizados en una escala de 0 a 100. 


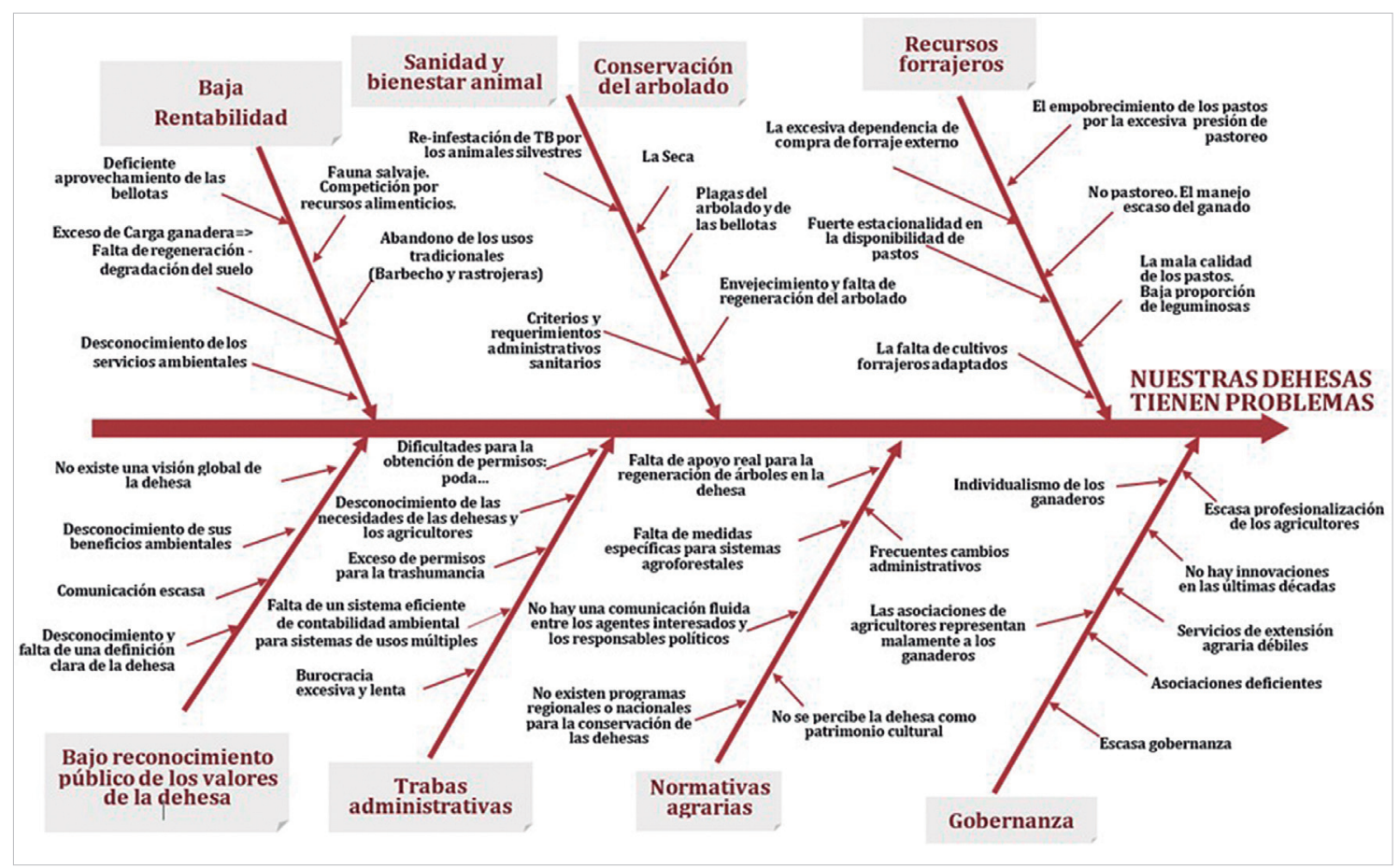

Figura 2. Diagrama de "raspa de pescado" o Ishikawa que resume las principales limitaciones determinadas por los participantes para las dehesas españolas, agrupadas por temáticas. La posición de las limitaciones y temáticas no refleja ninguna priorización.

poder generalizar su utilización y para mejorar su aplicación técnica en condiciones de explotaciones reales (Tab. 1). Aunque la mayoría de agentes interesados reconocieron la importancia de los servicios ambientales proporcionados por las dehesas, demandaron principalmente una investigación enfocada a resolver sus problemas de gestión diaria y aumentar la productividad de las dehesas, con énfasis en:

1.- La fertilidad del suelo.

2.- La mejora de la productividad y calidad de sus pastos.

3.- La producción propia del forraje y la internalización del ciclo de materiales.

4.- Los regímenes de pastoreo.

5.- La regeneración del estrato arbóreo.

6.- La diversificación de las actividades de producción.

7.- Valoración de los diferentes productos.

De forma más genérica también se demandaron estudios sobre las necesidades más críticas de la ganadería extensiva para ser más competitivos y rentables. La mayoría de los participantes estuvieron de acuerdo en que, mediante el aumento de la rentabilidad de las explotaciones de la dehesa, muchos de los retos existentes tendrían una fácil solución, ya que los agricultores invertirían más en sus explotaciones. El pago de los servicios ambientales prestados por las dehesas las haría más rentables, pero la mayoría de participantes fueron muy escépticos en cuanto al es- 
tablecimiento de este pago en un futuro cercano. También se señaló que la presencia de Altos Valores Naturales en sus dehesas tiene consecuencias perjudiciales para su economía, ya que tienen más limitaciones ambientales impuestas por las agencias de Medio Ambiente.

Tabla 1. Las innovaciones propuestas más demandadas por los actores interesados ( $\mathrm{n}=100) \mathrm{y}$ posteriormente priorizadas a través de encuestas a los mismos actores interesados ( $\mathrm{n}=43$ encuestas).

\begin{tabular}{|c|c|c|}
\hline & INNOVACIONES PROPUESTAS & $\begin{array}{l}\text { RANKING } \\
\quad(0-100)\end{array}$ \\
\hline \multirow{8}{*}{$\begin{array}{l}\text { Protección del arbolado } \\
\text { Valor de Prioridad }=\mathbf{1 0 0 . 0}\end{array}$} & $\begin{array}{l}\text { Prevención de la muerte súbita de las encinas y/u } \\
\text { otras quercíneas (La Seca) }\end{array}$ & 100.0 \\
\hline & Regeneración del arbolado: arbustos de vivero & 86.0 \\
\hline & $\begin{array}{l}\text { Regeneración del arbolado: protectores espinosos } \\
\text { artificiales }\end{array}$ & 66.7 \\
\hline & Regeneración del arbolado: plantas micorrizadas & 65.6 \\
\hline & $\begin{array}{l}\text { Regeneración del arbolado: repelentes químicos y } \\
\text { naturales }\end{array}$ & 40.9 \\
\hline & Cambiando el bosque denso a dehesas & 36.5 \\
\hline & $\begin{array}{l}\text { Tratamientos curativos para la muerte súbita de la } \\
\text { encina }\end{array}$ & 28.0 \\
\hline & Otras plagas y enfermedades & 25.8 \\
\hline \multirow{5}{*}{$\begin{array}{l}\text { Manejo pastoral } \\
\text { Valor de Prioridad }=71.4\end{array}$} & Pastoreo rotacional intensivo & 55.5 \\
\hline & Pastoreo de precisión- Instalaciones & 39.9 \\
\hline & Redileo del ganado & 38.9 \\
\hline & Pastoreo con collar GPS & 36.8 \\
\hline & Trashumancia & 35.3 \\
\hline \multirow{8}{*}{$\begin{array}{l}\text { Autonomía de forraje } \\
\text { y mejora de pastos } \\
\text { Valor de Prioridad }=66.9\end{array}$} & Siembras de pastos ricos en leguminosas & 63.0 \\
\hline & & \\
\hline & Cultivos Forrajeros: triticale de doble aptitud & 43.1 \\
\hline & Cultivos Forrajeros: leguminosas & 37.8 \\
\hline & Bancos forrajeros: arbustivos & 36.5 \\
\hline & Fertilización & 35.1 \\
\hline & Cultivos Forrajeros: variedades locales & 27.8 \\
\hline & $\begin{array}{l}\text { Cultivos Forrajeros: selección de las mejores } \\
\text { adaptadas a la sombra del árbol }\end{array}$ & 27.2 \\
\hline Protección del suelo y fertilidad & $\begin{array}{l}\text { Incremento de la materia orgánica del suelo por } \\
\text { pastoreo }\end{array}$ & 38.7 \\
\hline Valor de Prioridad $=53.7$ & $\begin{array}{l}\text { Incremento de la materia orgánica del suelo por } \\
\text { cultivo }\end{array}$ & 28.9 \\
\hline
\end{tabular}


(Viene de pág. anterior)

\begin{tabular}{|c|c|c|}
\hline & \\
\hline & INNOVACIONES PROPUESTAS & $\begin{array}{c}\text { RANKING } \\
(0-100)\end{array}$ \\
\hline & $\begin{array}{l}\text { Incremento de la materia orgánica del suelo con } \\
\text { astillas de madera (procedente de podas) }\end{array}$ & 25.6 \\
\hline \multirow{5}{*}{$\begin{array}{l}\text { Mercado } \\
\text { Valor de Prioridad = } 53.5\end{array}$} & Marca Dehesa & 42.3 \\
\hline & Producción orgánica & 33.6 \\
\hline & Calidad de la comida - Calidad del forraje & 27.0 \\
\hline & Contabilidad Ambiental - Pagos servicios públicos & 26.3 \\
\hline & Turismo & 25.5 \\
\hline \multirow{4}{*}{$\begin{array}{l}\text { Servicios ambientales } \\
\text { Valor de Prioridad = } 52.7\end{array}$} & Biodiversidad & 37.1 \\
\hline & Paisaje - Cultura & 36.2 \\
\hline & Secuestro de carbono & 26.6 \\
\hline & Huella ecológica & 22.0 \\
\hline \multirow{6}{*}{$\begin{array}{l}\text { Nuevos productos } \\
\text { Valor de Prioridad }=46.8\end{array}$} & Valorización de la biomasa & 34.7 \\
\hline & Bellotas dulces para la alimentación humana & 32.0 \\
\hline & Servicios ambientales públicos & 30.8 \\
\hline & Miel / Setas & 26.8 \\
\hline & Plantas aromáticas y medicinales & 22.0 \\
\hline & Cría de aves de corral & 16.0 \\
\hline \multirow{6}{*}{$\begin{array}{l}\text { Productividad del ganado } \\
\text { Valor de Prioridad = } 36.2\end{array}$} & Selección genética de animales más dóciles & 24.8 \\
\hline & Control de la re-infestación con animales salvajes & 24.4 \\
\hline & Selección genética de animales más productivos & 24.1 \\
\hline & Fertilidad animal & 20.5 \\
\hline & Sanidad animal & 16.8 \\
\hline & Bienestar animal & 14.9 \\
\hline \multirow{5}{*}{$\begin{array}{l}\text { Productividad de los árboles } \\
\text { Valor de Prioridad = } 31.6\end{array}$} & Selección genética para producir más bellotas & 26.7 \\
\hline & Selección genética para encinas precoces & 21.5 \\
\hline & Esquemas de poda & 18.0 \\
\hline & Selección genética para producir bellotas dulces & 14.5 \\
\hline & Métodos de injerto & 10.7 \\
\hline \multirow{2}{*}{$\begin{array}{l}\text { Normativa } \\
\text { Valor de Prioridad }=\mathbf{2 8 . 0}\end{array}$} & Elegibilidad completa de dehesas & 16.8 \\
\hline & Pagos de la PAC para contratos de gestión & 15.5 \\
\hline \multirow{3}{*}{$\begin{array}{l}\text { Gobernanza } \\
\text { Valor de Prioridad = } 25.1\end{array}$} & Gobernanza y asociaciones & 17.4 \\
\hline & Percepción pública y valorización de las dehesas & 13.4 \\
\hline & Registro de dehesas y cartografía & 12.7 \\
\hline
\end{tabular}


A continuación se detallan algunos de los aspectos más discutidos y demandados por el GTD, y para los que mayor disponibilidad se mostró para posibles programas de investigación colaborativa.

Para aumentar la rentabilidad de las explotaciones de Dehesa se identificó como prioritario:

- Aumentar la productividad y calidad de los pastos, optimizando las fuentes de proteínas para la alimentación animal (evitar el binomio soja-maíz) y aumentar la autonomía forrajera de las explotaciones:

- Siembra de praderas de secano ricas en leguminosas que perduren (autosembradas) bajo las condiciones ecológicas, sombra y de pastoreo de las dehesas.

- Control biológico de plagas en la bellota a través de esquemas de pastoreo.

- Como alternativa a la poda o desmochado de los árboles, se plantea el uso de arbustos forrajeros que se utilizarán como fuente de forraje para los periodos de verano e invierno.

- Se debe recuperar el cultivo parcial en la dehesa para producción de forraje, seleccionando las especies forrajeras mejor adaptadas a la sombra.

- Incrementar la materia orgánica del suelo. Resulta de interés la experiencia portuguesa “Terra Prima”, que hizo posible el pago de primas por el secuestro de carbono con la siembra de pastos diversos ricos en leguminosas.

- Desarrollar un Sistema de Contabilidad Ambiental adecuado para sistemas complejos como las dehesas y otros ecosistemas agroforestales, en los que se incluye el valor de los servicios ambientales, tales como la captura de carbono, la biodiversidad, la calidad del agua, la estética del paisaje... El objetivo final es poder asociar los pagos recibidos a los servicios ambientales prestados.

- Crear la Marca Dehesa para una gran cantidad de alimentos de alta calidad y materiales producidos en la dehesa, y para hacer más visible a los consumidores la relación entre el producto, el sistema productivo y los servicios ambientales asociados al producto.

Para mejorar la regeneración y protección del arbolado se identificó como prioritario:

- El uso de protectores de plantas más eficaces y económicos, tales como el uso de arbustos espinosos o protectores espinosos artificiales.

- El uso de plántulas de Quercus inoculadas con trufa de Bohemia (Pisolithus tinctorius; un potente estimulador de raíces) podría mejorar la resistencia a la sequía estival.

- Explorar nuevos regímenes de poda para optimizar la producción de bellota. La importancia de la poda de árboles para la producción de bellota a corto, medio y largo plazo sigue siendo ampliamente desconocida. 
- Priorizar el estudio de la muerte súbita de la encinas y/u otras quercíneas ("seca") en los Centros y Programas de Investigación Forestal en el suroeste de la Península Ibérica. Es necesario el análisis experimental de la eficacia de productos químicos como el dióxido de cloro y fosfitos como pulverizaciones foliares, y del encalado del suelo.

Para mejorar el aprovechamiento de los recursos forrajeros, se identificaron posibles mejoras en el manejo pastoral:

- Vinculación de la carga animal a la disponibilidad de recursos forrajeros y no a la normativa de subvenciones.

- Recuperar algunas prácticas tradicionales como el redileo del ganado para mejorar la calidad de suelos y pastos.

- Pastoreo holístico (ciclos de rotación cortos e intensos) y Pastoreo de precisión mediante el diseño de calendarios para la ubicación de utilidades (suplemento forrajero, abrevaderos, sales minerales).

- Pastoreo con GPS para ayudar al pastoreo de precisión, la protección de la regeneración de árboles, el control de la re-infestación del ganado por animales salvajes, la vigilancia de la salud del ganado, etc. Aunque la aplicación de GPS para seguir y analizar la actividad de los animales está técnicamente resuelta, sigue siendo un gran desafío la aplicación de estímulos negativos/positivos para conducir el comportamiento animal.

Para mejorar la producción animal se identificó como prioritario:

- Utilización de las razas ganaderas mejor adaptadas a la estacionalidad de pastos, las más dóciles, las más cuidadosas con el arbolado (menos ramoneadoras).

- Diversificación de la ganadería. Se mencionan gansos, pavos y ciervos.

- Mejora de los índices de fertilidad, por ejemplo, evaluando la calidad del semen de sementales).

- Para reducir la carga parasitaria es necesario proporcionar al ganado agua de alta calidad a través de comederos móviles y evitar el acceso directo de los animales a charcas por su potencial impacto negativo en la salud animal.

- Reducir la re-infestación de tuberculosis por los animales salvajes en los puntos de abastecimiento de agua y en los puntos de provisión supletoria de piensos.

También se identificó como necesario la Diversificación de la Producción valorando productos nuevos y tradicionales de las dehesas:

- Usando genotipos de encina de bellota dulce (con bajo contenido de taninos) para producir alimentos de alta calidad tales como harinas sin gluten, tortas, helados, horchata (leche vegetal), aceites ricos en ácidos grasos insaturados....

- Selvicultura para la producción de setas comestibles como Boletus edulis, Amanita caesarea y A. ponderosa, Terfezia arenaria..., comunes en las dehesas. 
— Valorización de la leña y subproductos como las ramas podadas y el sotobosque arbustivo.

- Gestión y valoración de otros productos de la dehesa, como la miel (procedente de mela de la encina), corcho manufacturado, plantas aromáticas y medicinales, taninos naturales...

Finalmente se señalaron varias necesidades en el ámbito de la Normativas, Gobernanza y Formación:

- Regulaciones específicas para las dehesas y otros sistemas agroforestales.

- Simplificación de la burocracia y centralización de la administración de la dehesa.

- Elegibilidad completa de las dehesas y pagos del pilar I de la PAC.

- Pagos públicos por el Alto Valor Natural y Cultural de la dehesa.

- Se destacó la necesidad de una federación nacional de agricultores de la dehesa. Recientemente se ha creado FEDEHESA (Federación Española de la Propietarios y Gestores de Dehesa).

- Creación de un programa público de investigación de la dehesa y otros sistemas silvopastorales.

\section{Conclusiones}

En esta primera fase del proyecto tuvimos como objetivo;

1.- Presentar el proyecto a los posibles agentes interesados.

2.- Conocer la percepción de las partes interesadas de los sistemas agroforestales, y más concretamente los de Alto Valor Natural y Cultural.

3.- Compilar una lista priorizada de las innovaciones que podrían ayudar a mejorar la rentabilidad y la sostenibilidad de las dehesas.

4.- Conformar una Red de Investigación y Desarrollo Participativo (RIDP) para poner a prueba las innovaciones propuestas y difundir los resultados. Esto se ha logrado gracias a la amplia y activa participación de los agentes interesados en la primera sesión pública, a las respuestas a los cuestionarios y a los nuevos comentarios de los miembros de la RIDP sobre el primer borrador de este informe.

La percepción de los principales actores de la dehesa es que éstas pueden producir diversos productos de alta calidad y proporcionar servicios ambientales importantes. Sin embargo, la baja rentabilidad actual de las explotaciones, asociada a la progresiva degradación de los suelos, los pastos y el estrato arbóreo, pone en peligro la persistencia de este Sistema de Alto Valor Natural. Para superar estas dificultades, el GTD ha identificado una larga lista de innovaciones que eventualmente podrían mejorar la sostenibilidad económica, ecológica y social de las dehesas. Entre las innovaciones más demandadas cabe destacar las referentes a la regeneración del arbolado, implantación y gestión de pastos permanentes ricos en legumi- 
nosas, cultivos forrajeros adaptados a la sombra, nuevas tecnologías de apoyo al pastoreo racional y para optimizar el aprovechamiento de los recursos forrajeros internos, y explorar la posibilidad de crear una marca para los productos de la dehesa.

Además, los participantes reclamaron una plataforma para el intercambio de conocimientos, experiencias e ideas, para una mejor gobernanza y para adaptar mejor las múltiples prácticas de gestión en la dehesa. Así se podrá conseguir de manera sostenible la prestación de servicios ambientales públicos y la conservación del legado natural y cultural.

\section{Bibliografía}

Asner, G.P., Elmore, A.J., Olander, L.P., Martin, R.E., Harris, A.T., 2004. Grazing systems, ecosystem responses, and global change. Annu. Rev. Env. Resour. 29, 261-299. https:// doi.org/10.1146/annurev.energy.29.062403.102142

den Herder, M., Moreno, G., Mosquera-Losada, R.M., Palma, J.H.N., Sidiropoulou, A., Santiago Freijanes, J.J., Crous-Duran, J., Paulo, J.A., Tomé, M., Pantera, A., Papanastasis, V.P., Mantzanas, K., Pachana, P., Papadopoulos, A., Plieninger, T., Burgess, P.J., 2016. Current extent and stratification of agroforestry in the European Union. Agr. Ecosyst. Environ. 241, 121-132. https://doi.org/10.1016/j.agee.2017.03.005

Eichhorn, M.P., Paris, P., Herzog, F., Incoll, L.D., Liagre, F., Mantzanas, K., Mayus, M., Moreno, G., Papanastasis, V.P., Pilbeam, D.J., Pisanelli, A., Dupraz, C., 2006. Silvoarable systems in Europe - past, present and future prospects. Agrofor Syst. 67(1), 29-50. https://doi.org/10.1007/s10457-005-1111-7

Gaspar, P., Mesías, F.J., Escribano, M., Pulido, F., 2009. Sustainability in Spanish extensive farms (Dehesas): an economic and management indicator-based evaluation. Rangeland Ecol. Manag. 62(2), 153-162. https://doi.org/10.2111/07-135.1

Jose, S., 2009. Agroforestry for ecosystem services and environmental benefits: An overview. Agroforest. Syst. 76, 1-10. https://doi.org/10.1007/s10457-009-9229-7

Moreno, G., Pulido, F.J., 2009. The functioning, management, and persistence of dehesas. In: Riguero-Rodríguez, A., Mosquera-Losada, M.R., McAdam, J. (eds.), Agroforestry Systems in Europe: Current Status and Future Prospects. Springer, Dordrecht.

Pulido, F., García, E., Obrador, J.J., Moreno, G., 2010. Multiple pathways for tree regeneration in anthropogenic savannas: incorporating biotic and abiotic drivers into management schemes. J. Appl. Ecol. 47(6), 1272-1281. https://doi.org/10.1111/j.13652664.2010.01865.x

Ramankutty, N., Evan, A.T., Mongreda, C., Foley, J.A., 2008. Farming the planet: 1. Geographic distribution of global agricultural lands in the year 2000. Global Biogeochem. Cy. 22(1), 1-19. https://doi.org/10.1029/2007GB002952

Rigueiro-Rodríguez, A., McAdam, J., Mosquera-Losada, M.R., 2009. Agroforestry in Europe: Current Status and Future Prospects. Springer, Dordrecht. https://doi.org/ 10.1007/978-1-4020-8272-6

Rundel, P.W., 1998. Landscape Disturbance in Mediterranean-Type Ecosystems: An Overview. In: Rundel, P.W., Montenegro, G., Jaksic, F.M. (eds.) Ecological Studies 136: 
Landscape Disturbance and Biodiversity in Mediterranean-Type Ecosystems, SpringerVerlag Berlin, Heidelberg. https://doi.org/10.1007/978-3-662-03543-6_1

Zomer, R.J., Trabucco, A., Coe, R., Place, F., van Noordwijk, M., Xu, J.C., 2014. Trees on farms: an update and reanalysis of agroforestry's global extent and socio-ecological characteristics. Working Paper 179. Bogor, Indonesia: World Agroforestry Centre (ICRAF) Southeast Asia Regional Program. https://oi.org/10.5716/WP14064.pdf 
